\title{
Design, Manufacturing, and Commissioning of BIRCAM (Bootes InfraRed CAMera)
}

\author{
Alberto Riva, ${ }^{1}$ Paolo Conconi, ${ }^{2}$ Alberto Javier Castro-Tirado, ${ }^{3}$ Filippo Zerbi, ${ }^{2}$ \\ Ronan Cunniffe, ${ }^{4}$ Martin Jelinek, ${ }^{3}$ and Stanislav Vìtek ${ }^{5}$
}

${ }^{1}$ INAF-Osservatorio Astronomico di Torino, Strada Osservatorio, 20, 10025 Pino Torinese, Italy

${ }^{2}$ INAF-Osservatorio Astronomico di Brera, Via Emilio Bianchi, 46, 23807 Merate, Italy

${ }^{3}$ IAA-Instituto de Astrofisica de Andalucia (CSIC), Camino Bajo de Huétor, 50, 18008 Granada, Spain

${ }^{4}$ Blackrock Castle Observatory (Cork Institute of Technology), Castle Road, Mahon, Cork, Ireland

${ }^{5}$ Department of Radio Engineering, Faculty of Electrical Engineering, Czech Technical University in Prague, Technickà, 2, 166 27, Praha, Czech Republic

Correspondence should be addressed to Alberto Riva, riva@oato.inaf.it

Received 30 June 2009; Accepted 23 November 2009

Academic Editor: Joshua S. Bloom

Copyright ( $) 2010$ Alberto Riva et al. This is an open access article distributed under the Creative Commons Attribution License, which permits unrestricted use, distribution, and reproduction in any medium, provided the original work is properly cited.

This paper covers the various aspect of design, manufacturing and commissioning of the infrared camera BIRCAM, installed at BOOTES-IR, the $60 \mathrm{~cm}$ robotic infrared telescope at Sierra Nevada Observatory (OSN, Granada, Spain). We describe how we achieved a quality astronomical image, moving from the scientific requirements.

\section{Introduction}

Fast pointing robotic telescopes for transient detections have decades of tradition in astronomy. However, the first successful attempt to extend their coverage to the IR band is REM (Rapid Eye Mount) installed in 2003 at La Silla Observatory and currently still in operation. REM was conceived as the ground-based complement of the satellite SWIFT $[1,2]$.

Well beyond its original purposes, REM is today serving the Italian Community as a long-term monitoring facility for variable stars, AGNs, Supernovae and many other types of programs that need IR observations. Moreover, it invalidated the equation "IR equal to large telescope" and opened to a number of projects such as BOOTES-IR with its infrared camera BIRCAM.

BIRCAM is an astronomical infrared camera, installed on the Spanish BOOTES-IR telescope (Observatorio de Sierra Nevada, Granada, Spain) and is designed for the Gamma Ray Burst afterglow observations, as the original REMIR camera installed on REM.

\section{General Overview of BIRCAM}

BIRCAM (Bootes-IR CAMera) is a near infrared camera working in the 1-2.3 microns wavelength range. It is installed at one of the Nasmyth foci of the BOOTES-IR.

The design of the camera is strictly joined with its telescope. Indeed, they have as primary objective the acquisition of images of the GRB early afterglows.

Observations at infrared wavelengths are of great importance in astronomy. One of the most known advantages concerns the observation of the distant universe. Due to red-shift, distant objects have their spectral features moved toward higher wavelengths. If one wants to compare the rest-spectrum of a nearby galaxy in the $V$ band (500600 nanometers) with a galaxy at $z=3$, he will have to observe the latter between 2000 and 2400 nanometers, that is, in the infrared region. Furthermore if the Lyman break (912 Angstroms) is redshifted to the upper limit of the visible region $(z \sim 7-8)$, the object is detectable only in the infrared.

Further advantage of the infrared band with respect to the visible is the reduced absorption of the radiation by the 


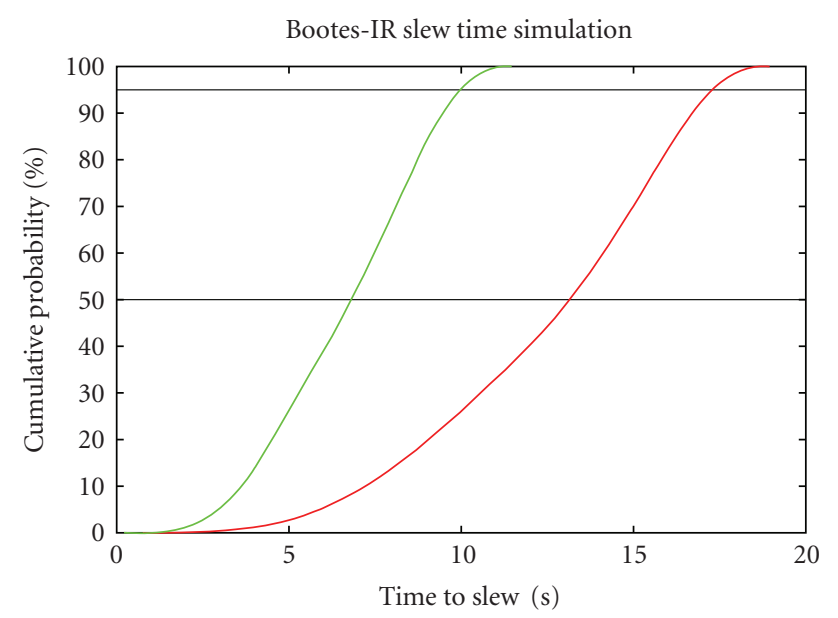

$-a=2 \mathrm{deg} / \mathrm{s} 2$

$a=8 \mathrm{deg} / \mathrm{s} 2$

FIGURE 1: Simulation of slews times for Bootes-IR.

interstellar medium. Indeed the absorption in the classical $V$ band is about one order of magnitude greater than the absorption in the $K$ band. Hence any observation of sources in dusty areas such as galactic centers, clouds, and so forth. benefits of being carried out in infrared instead of visible.

Moreover, every astronomical source for which the thermal blackbody emission is relevant, if cold, will emit in the infrared band. These objects are typically brown dwarfs, planets, protostars, low mass stars.

The telescope-camera system is similar to the REM project, installed in Chile (La Silla) [3]. Both telescopes are in communication with the mission satellite SWIFT [4], that is, able to detect and give the position of a GRB within 10 seconds with a precision of 3 arcminutes. They also follow up other sources of trigger such as INTEGRAL and GLAST.

The REM telescope is able to point the source in 3045 seconds with a field of 10 arcminutes squared. Instead, BOOTES-IR is able to point the source in 10-20 seconds with a field of 12 arcminutes squared.

The coordination between the satellite and the two telescopes located in different parts of the earth leads to the coverage of large number of the GRB within the first minute after their detection. Both telescopes are provided with a simultaneous covering of visible and near infrared wavelength range.

Two other currently active near infrared instruments in the GRB detection are: PAIRITEL [5] and GROND [6]. BOOTES-IR presents two main advantages with respect to those two instruments: the position and the speed. Indeed BOOTES-IR is situated at more than $70^{\circ}$ in longitude compared to these two instruments. This allows observations of GRB when it is day over PAIRITEL and GROND. Regarding the speed, BOOTES-IR is faster than GROND, and this fact is essential in the observation of the first minutes after the SWIFT trigger. This can lead to a detection for faint afterglows or a catching for eventual flashes.

In order to understand how the camera has been designed we need to briefly describe the characteristics of the
TABLE 1: Basic parameters for $1024 \times 1024$ pixels HgCdTe, Hawaii1, Rockwell detector.

\begin{tabular}{lcc}
\hline Parameter & Measured value & Units \\
\hline Die size & $<400$ & $\mathrm{~mm}^{2}$ \\
Integration capacity & $1.0 \times 10^{5}$ & carriers \\
Integration capacitance & $18-35$ & $\mathrm{fF}$ \\
Signal Conversion Gain & $3.6-4.8$ & ${\mathrm{~V} / \mathrm{e}^{-}}^{-}$ \\
Output Signal Excursion & $0.4-1$ & $\mathrm{~V}$ \\
Minimum Read Noise & $<10$ & $\mathrm{e}^{-}$ \\
Dynamic Range & $\mathrm{Up}$ to $10^{4}$ & $\mathrm{~V} / \mathrm{V}$ \\
Maximum data rate & $<1$ & $\mathrm{MHz}$ \\
Maximum Slew rate & 400 & $\mathrm{nsec}$ \\
Peak D* @ min Qb & $>1 \times 10^{14}$ & $\mathrm{Jones}$ \\
Power dissipation & $\leq 1$ & $\mathrm{~mW}$ \\
\hline
\end{tabular}

telescope. Built by ASTELCO GmBH (Munich, Germany), this telescope is a $0.6 \mathrm{~m}$ Ritchey-Chrétien working with a focal number of $f / 8$. Its direct drives were designed to allow it to slew at a maximum acceleration of $8^{\circ}$ per second, with a maximum speed of $20^{\circ}$ per second, being capable of pointing any part of the sky within 20 seconds. In other words, $50 \%$ of slews ends within 6.8 seconds and 95\% slews within 10 seconds, see Figure 1. It has two Nasmyth foci, one of which is occupied by both the near infrared and optical cameras. Currently the second Nasmyth is not used, although future instrumentation is foreseen.

The infrared camera is equipped with a $1024 \times 1024$ pixels HgCdTe detector (Hawaii-1, Rocwell), sensible from 0.9 to $2.5 \mu \mathrm{m}$. It has a pixel size of $18.5 \mu \mathrm{m}$, four outputs, $<10 \mathrm{e}^{-}$ read noise, up to $1 \mathrm{MHz}$ data rate per output, low power dissipation, $<0.1 \mathrm{e}^{-} / \mathrm{sec}$ dark current at $78 \mathrm{~K}, 3.4$ to $6.8 \mu \mathrm{V} / \mathrm{e}-$ signal conversion gain. In Table 1 we report a summary of relevant detector data. The controller is a Skytech CTRA1700 CCD with a 4-channel video board customised for infra-red (CDS-A100_2.00).

The pixel scale is $0.7^{\prime \prime}$, providing a field of view of $12^{\prime} \times 12^{\prime}$. Dithering of the images is achieved by using a rotating tilted-window (dithering wedge), placed in front of the camera, which displaces the image in a circular pattern. This allows us to later correct the sky background present in NIR images.

The electronic system that is responsible to acquire photons and translate them into readable signals has been designed and built by SKYTECH Electronic Technologies (La Spezia, Italy). The cooling down of the detector, based on a bath of liquid nitrogen, and the cryomechanical system has been designed and built by the Italian company RIAL Vacuum S.p.A. (Parma, Italy).

The optical path was designed in cooperation by P. Conconi and A. Riva, elements were procured from two companies, OptoTL (Saint-Petersburg, Russia), and Laboratorio Ottico Colombo (Rovagnate, Italy).

Finally, the integration of each subsystem and the characterization of the optomechanical properties of the camera was carried out under responsibility of A. Riva in the framework of gOlem laboratories (INAF, Merate, Italy). 


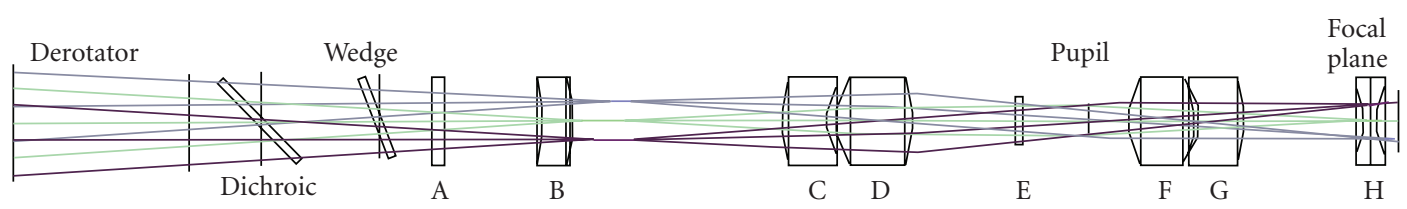

FIgURE 2: Optical layout of BIRCAM.

TABLE 2: Basic data of each lens. Note that the radii are expressed positive or negative because referred to a common coordinate system.

\begin{tabular}{|c|c|c|c|c|c|}
\hline Name & Material & Thickness (mm) & Radius $1(\mathrm{~mm})$ & Radius $2(\mathrm{~mm})$ & Diameter $(\mathrm{mm})$ \\
\hline Entrance window (A) & $\mathrm{CaF}_{2}$ & 6.00 & infinite & Infinite & 60.00 \\
\hline First lens (B) & SFTM-16 & 14.00 & 86.04 & 69.05 & 50.80 \\
\hline 1st collimator lens $(\mathrm{C})$ & SFTM-16 & 20.00 & -69.05 & -28.00 & 50.80 \\
\hline 2nd collimator lens (D) & $\mathrm{CaF}_{2}$ & 35.00 & -32.68 & 59.64 & 50.80 \\
\hline 1st camera lens $(\mathrm{F})$ & $\mathrm{CaF}_{2}$ & 30.00 & -38.76 & 38.76 & 50.80 \\
\hline 2nd camera lens $(G)$ & SFTM-16 & 20.00 & 28.00 & 69.05 & 50.80 \\
\hline Field flattener (E) & $\mathrm{CaF}_{2}$ & 6.00 & 37.00 & -37.00 & 50.80 \\
\hline
\end{tabular}

The following paragraphs contain a description of some interesting issues related to the various phases of the project.

\section{Optical Design: The Answer to the Scientific Requirements}

The optical system of the camera is completely transmissive, composed by optical elements of two materials: the CaF2 (calcium fluoride) as crown, and SFTM-16 (O'Hara glass) as flint.

In Figure 2, we show the final raytracing of the camera. The first surface in the figure represents the Derotator, a mechanical flange that compensates the field rotation at Nasmyth focus. It is essentially the mechanical interface that the telescope offers to both the visible and near infrared cameras. Between the derotator and the first element of the camera (A), there are other two optical elements: the Dichroic with the function of separate the visible light from the infrared, and the Wedge that rotate along the optical axis with the function to move the viewed field at different positions on the detector. The function of the wedge is basically the dithering of the image to increase the signal to noise ratio. [7]

The first optical element of the camera is the entrance window (labelled with letter A). It is a CaF2 disk of $60 \mathrm{~mm}$ of diameter and $6 \mathrm{~mm}$ of thickness. Before reaching the first focal position, the beam encounters the first SFTM-16 lens (B). This lens acts like a FPAL (Focal Plane Adaption Lens), and reduces the curvature of the field and the astigmatism. It is designed in order to simplify the design of the collimator. The elements labelled with $\mathrm{C}$ and D are the crown/flint couple that compose the collimator. Indeed their function is to form a parallel beam where the filter position (E) and the pupil stop are located.

After the pupil, there is another couple of crown/flint glasses ( $F$ and $\mathrm{G}$ ) with the function of reforming a focalized beam, usually called camera. The last element before the focal plane is the field flattener $(\mathrm{H})$. The final equivalent focal ratio of the system is 9.35. In Table 2 we report the basic data of each lens belonging to BIRCAM.

Following the experience of REMIR camera (the Infrared camera on REM telescope) we adopted a unconventional solution for the size of the lenses. Indeed looking at the shape of most of the lenses, specially the doublets of collimator and camera, it is possible to notice that they are thicker than the lenses usually adopted in astronomical instrumentation. The choice of such large thickness is one of the most interesting features of this design. All the lenses (from B to $\mathrm{H}$ in Figure 2) are placed in a optical bench with a $V$ shaped machining (see Figure 6). The combination between the precision in the cylindrical shape of each lens and the precision in the milling of the $V$, is a guarantee on the precision on the aligning of each lens with respect to the optical bench, and in second analysis it guarantees a good aligning between each lens.

This feature is strongly helpful in cryogenic system because the cool down process, with its related differential shrinking between each material, does not affect the global alignment of the optical train. The only attention to be paid during the manufacturing of each lens is the over-sizing of each element proportional to its coefficient of thermal expansion (CTE).

3.1. Dichroic. Special attention has been put on the design and procurement of the dichroic. It does not belong to the camera structure but it has strong effects on the final image on the detector. It is essentially a CaF2 disk tilted by $45^{\circ}$ along the optical axis. Its function is to separate the electromagnetic spectrum coming from the telescope into two beams. It operates both in transmission and in reflection. Due to the special interferential coating, it is able to transmit the infrared radiation and reflect the visible one. The design of the dichroic coating was done via the FilmStar32 software.

Since the visible path is materialized after a reflection it is basically undistorted, on the other hand the infrared path crosses the dichroic and the wavefront is consequently modified. From the point of view of the infrared camera the 


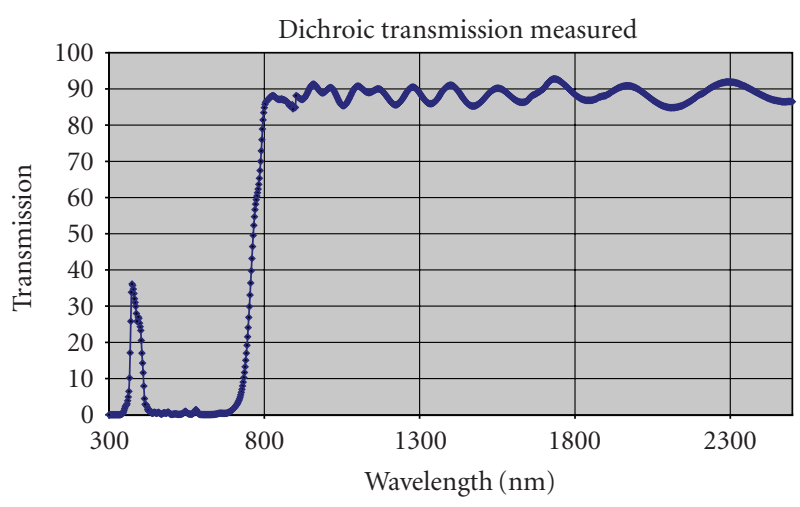

FIgURE 3: Transmission measured on final coating of the dichroic.

dichroic is essentially a plane parallel plated tilted by $45^{\circ}$ and that implies an introduction some amount of astigmatism distortion. Following the same reasoning and looking at Figure 2, it seems that the dithering wedge acts in the same way. This effect (smaller because introduced by an angle of $20^{\circ}$ ) appears along the direction perpendicular to the tilt axis, which rotates around the optical axis. The astigmatism introduced by the dichroic is instead greater and always displaced in the same direction. In order to correct the aberration introduced, the dichroic is designed with a back cylindrical surface (concave radius of $12800 \mathrm{~mm}$ ). The axis of the cylinder is parallel to the tilt axis.

In Figure 3 we show the final output of the dichroic coating transmission measured with a spectrophotometer available within gOlem laboratories (Merate, Italy). As requested by the final user, the transmission band starts around $800 \mathrm{~nm}$, in order to include the possibility of using inside the infrared camera an UKIRT z-filter filter (central wavelength $877 \mathrm{~nm}$, FWHM $95 \mathrm{~nm}$, cut-on $830 \mathrm{~nm}$, cut-off $925 \mathrm{~nm})$.

\section{Cryomechanical System}

One of the features of the Nasmyth focus, during a tracked observation, is the fact that the field observed by the telescope rotates around its central point. In Bootes-IR telescope case, the problem is resolved installing a mechanical system (a derotator) that counter-rotate the mechanical interface on the mounting flange of the Nasmyth focus. This fact allows BIRCAM camera, mounted on such flange, to point at the same field, avoiding any error introduced on the images and avoiding complicate calculation on the astrometry of each acquisition. The rotation of the camera is equivalent to the field rotation at the Nasmyth focus, compensating for the change of the parallactic angle during the observation. For a fast telescope-camera project, any loss of time implies a delay on the final image, and degradation on the scientific performances.

A peculiarity of this project consists in the cooling system. It is essentially based on liquid nitrogen. Liquid nitrogen offers the possibility to avoid any further mechanism loaded at the Nasmyth focus mounting. The price to be paid for this advantage consists in the complexity of the pipe scheme on

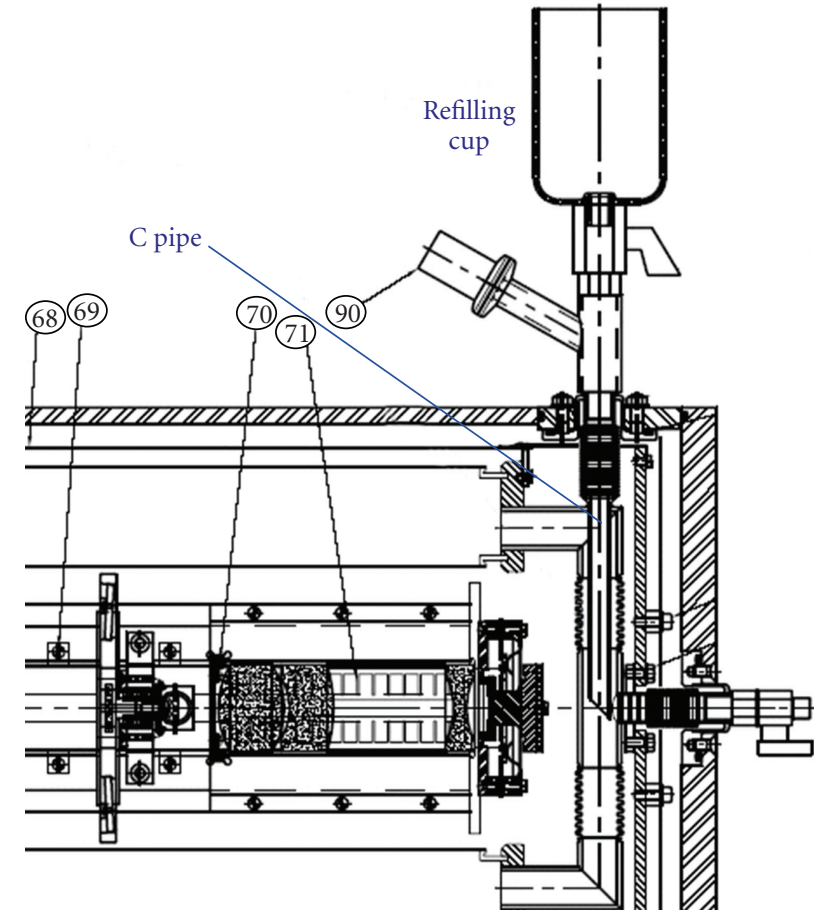

FIGURE 4: Scheme of the refilling mechanism in order to prevent leak of nitrogen during the derotation.

the loading part of the refilling system in order to compensate the rotation of the dewar, shown in Figure 4 and described below.

The camera is designed to be refilled manually every 24 hours, with the possibility of an upgrade with an automatic system. This choice fixes some constraints upon the mechanical design and production. The first and obvious implication is the presence of a tank for the storage of the liquid nitrogen. This tank is at cryogenic temperatures $(77 \sim 80 \mathrm{~K})$ and it has to be placed in a vacuum vessel in order to reduce the thermal contact and the condensation of air in order to maximize the duration of the liquid form of nitrogen, and hence the cooling power.

As described above it is almost impossible to have a tank completely insulated from air if it needs a feeding pipe. Another complication given by the presence of a tank of liquid nitrogen is related to the evaporation of the nitrogen itself. The volume ratio between the gas form and the liquid form of nitrogen is about 700, so it is strictly necessary to provide the tank with a venting system. This system is complicated by the rotation of the telescope. It implies that the gaseous form of nitrogen needs to have a continuous outlet regardless of the orientation of the tank. The best solution for these cases is to have a tank with the volume doubled with respect to the filling capacity, with several outlets that stand on top of the liquid in every position of the camera. It also implies to have a system that closes the outlet that time by time stands on the bottom of the liquid and would leak nitrogen.

The solution adopted for this camera is a tank with a Cshaped pipe with outlet on top and bottom of the tank. The $\mathrm{C}$ present a valve on the rotational axis of the tank, which 


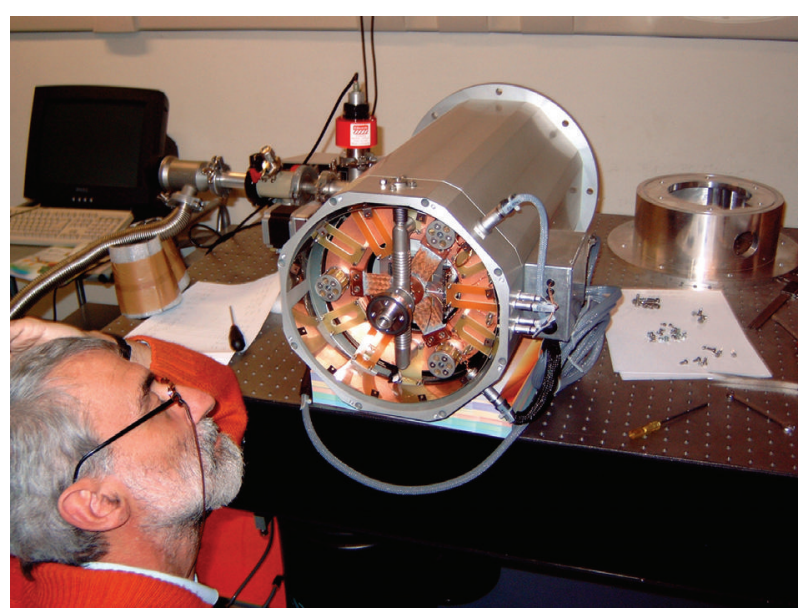

FIgure 5: BIRCAM electronic, fiberglass, C-pipe during the assembling phase.

helps during the refilling operation, because once the tank is half-filled, the exceeding nitrogen exits the tank through the valve pointing out the end of refilling.

As mentioned before, there is another complication due to the refilling channel. In Figure 4 we show a representation of the tank scheme. It is possible to notice the refilling cup on the right with an asymmetric displacement respect to the rotational axis of the camera. The cup is connected to the axis of the tank trough a needle, that prevent any radial leak of liquid nitrogen once the camera is rotated and the cup is downside. Nevertheless, both the axial valve and the radial valve have a closing system. The overpressure due to the evaporation of the nitrogen is prevented through a valve, that gets the gas from the axial position.

\section{Integration}

The integration took place in gOlem facilities (Merate, Italy). Once the optical pieces, the mechanical pieces and the electronic pieces were delivered. Integration implied aligning of optical elements on the bench, characterization of the entire system under the cryo-mechanical point of view. At this stage the detector system was not part of the integration activities. This because, contractually, the camera did not include the delivery and the characterization of any scientific detector. The development of the control electronics was carried out through a multiplexer, a device that simulates the electrical scheme of the science detector. This caused problems in the verification of the accuracy of the alignment of the camera. Indeed, as described before, the optical train is designed in order to function at cryogenic temperature with infrared sources. This feature implies that the quality of the design (mechanical, optical, electronical and cryogenic) can be verified under these conditions only with the final detector.

Fortunately the multiplexer is sensitive to the visible light. Hence by means of a laser lighted in the train is possible to have an idea of the projection of a star source on the detector.

A further analysis of the system was performed through an interferometer available in our laboratories. We essentially put the camera as a single element in the nominal focal position of the instrument, having as output the global quality of the system. One of the limits of this way of measuring was in the power of the testing source, heavily absorbed by the number and the materials of the lenses of the camera.

The most important result of these tests was that the tolerances given by the system, and in particular by the shape of the lenses and the spacers between them, was highly within the limits of the goals set for this camera.

In following Figures 4 and 5 we show some snapshot of the integration phases.

\section{Tests}

During the integration phase various problems were encountered. The most important fact was related to the liquid nitrogen consumption. The initial calculation carried out by RIAL technicians, and the constraints about the total space available between the telescope and the dome, lead to the dimensioning of the capacity of the nitrogen tank in order to reach autonomy of 24 hours. In the early integration tests, this goal was fully accomplished as can be seen from Figure 7 . In successive characterizations tests, the performance of BIRCAM decreased until a minimum autonomy of 12 hours.

The investigation of the problem was conducted through successive steps. The philosophy in setting up the successive tests was aimed in excluding step by step any possible source of the problem.

We will not describe here all tests done in one year of trials. We will summarize below the most interesting.

(i) Leak test: we measured any possible vacuum leak with specific helium-based leak-detector. This type of dewar is sensitive to wrong positioning of sealing orings.

(ii) Weight test: one of the probable cause of the low autonomy could be the formation of ice inside the tank. The measure of weight of BIRCAM during the first filling operation shown that the real load of nitrogen was compliant with the design.

(iii) Extra radiation: one of the possible agents that increased the consumption could be an increased amount of radiation going in the camera through the entrance window. The measure with the window and with an aluminium disk was substantially the same.

(iv) Wrong shielding: some modification on original radiation shield suggested the possibility that the new configuration increased the radiation between the external and internal part of dewar.

(v) Extra current: the multiplexer adopted for electronic test was operated in a different configuration with respect to the science detector, increasing the nitrogen consumption.

(vi) Thermal simulation: we simulated via Finite Elements Analysis (FEA) software the consequence of an unwanted thermal bridge between the dewar and the shield. 


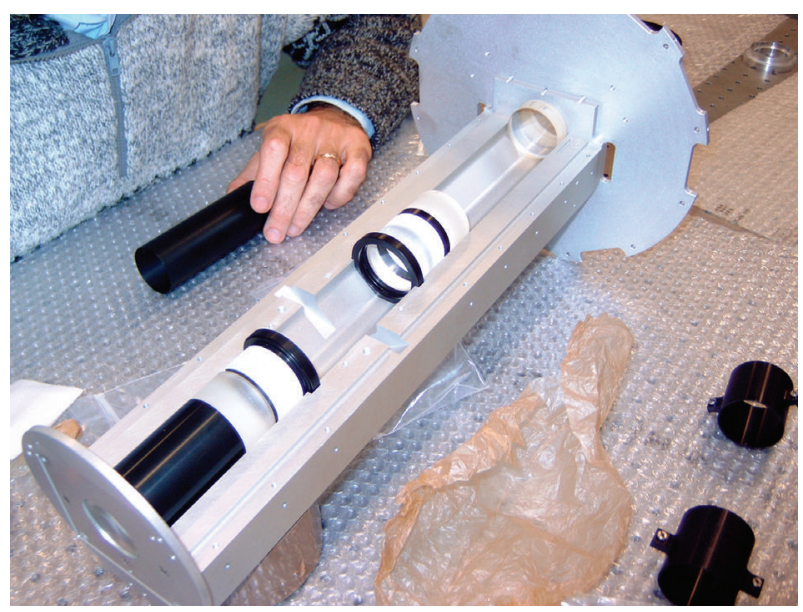

Figure 6: Assembling of lenses and spacers on the V-cave of the optical bench.

(vii) Shield configuration: we added and removed reflective layers of thermal shield in order to evaluate the radiation contribution.

(viii) Tank pressure: we tried different values of pressure inside the nitrogen tank in order to evaluate if nitrogen vapours could contribute to the global thermal balance.

All tests helped in understanding and improving the performances, but at the current date the 24-hour limits is not reached. So improvements were achieved but not allowing to solve the main problem. We stated that the problem stands in the dimensioning of the tank. Future upgrades (now under study) could be developed expanding the tank with an additional stage. We show in Figure 8 as example the temperature and pressure graphs for one of the intermediate test.

\section{Commissioning}

After the phase of testing and setting up, the camera was accepted and delivered to Granada (IAA) Laboratories to undergo the phase of the final integration. In this phase, carried out by R. Cunniffe and A. Riva, the substitution of the multiplexer with the final scientific detector, the 1024 $\times 1024$ pixels HgCdTe, Hawaii (Rockwell, Thousand Oaks, CA, USA) was operated. This task was completed using the clean room and the optical equipment of the IAA. This allowed to perform a further test in visible light, using the residual sensitivity to visible light (laser beam) of the Scientific Detector. In addition we performed an infrared light test, using a pinhole placed in front of the camera, with a common candle as light source.

After a obvious phase of tuning of the electronic readout devices, the camera, in April 2008 was able to be installed at the Nasmyth focus of BOOTES-IR. This stage was dedicated to the setup of the Linux software dedicated to this experiment. Indeed the SKYTECH company provided only a Windows version with the capability to read only one

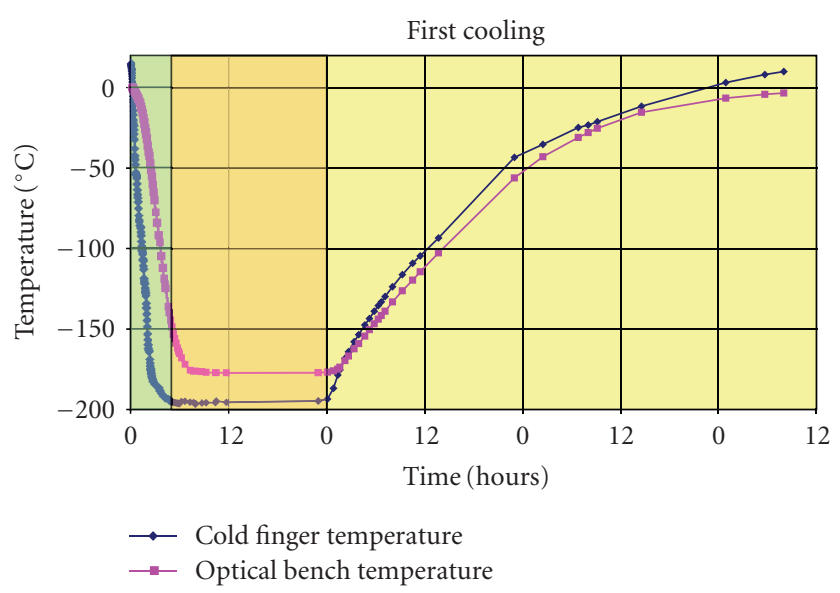

FIGURE 7: Graph of the temperatures during a complete cycle of cooling down and warm up on BIRCAM. Cold finger is located behind the detector. Optical bench sensor is located next B lens in Figure 2. The first part (first band, about 5 hours) is the cooling down phase, the second part (second band, until first 24 hours) is the stationary cold phase, the last one (about two days and half) is the warm up phase.

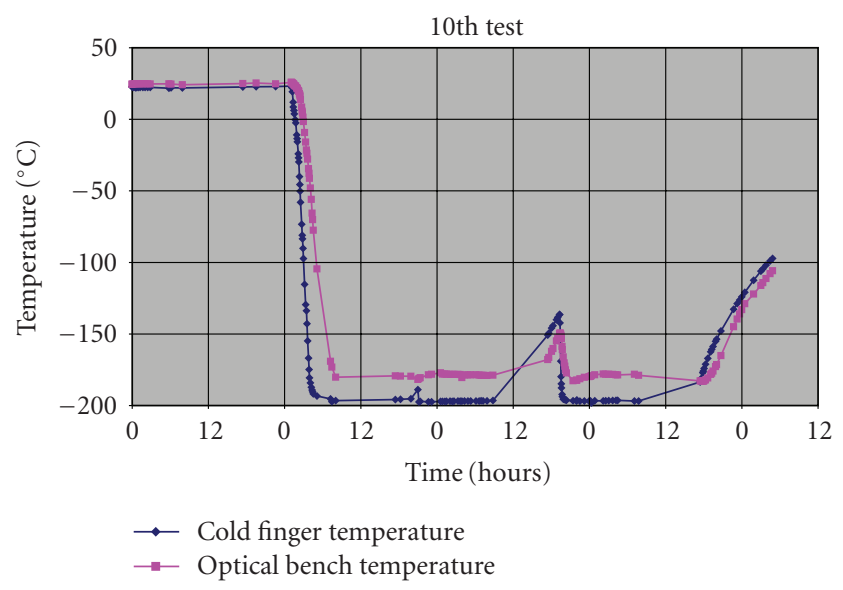

Figure 8: Temperature graph of a laboratory intermediate test of endurance. After the first cooling down, the camera was maintained at low temperature with successive refilling. The little bump in the middle of the plateau reflects a lack of refilling (night time).

of the four quadrant in a single exposure. The final tuning of Linux is still to be completed, however the 16th October 2008 the BIRCAM + BOOTES-IR teams obtained the first scientific light in J band of the well known M82 galaxy, shown in Figure 10.

The first light images were taken with an experimental software package and have shown that many things have to be solved yet. The estimate of a limiting magnitude of one of these images is $J \sim 16$ for a $3 \times 60$ seconds exposures.

\section{Lessons Learned}

As stated at the beginning the BIRCAM project was followed end-to-end from the design to the final integration and 


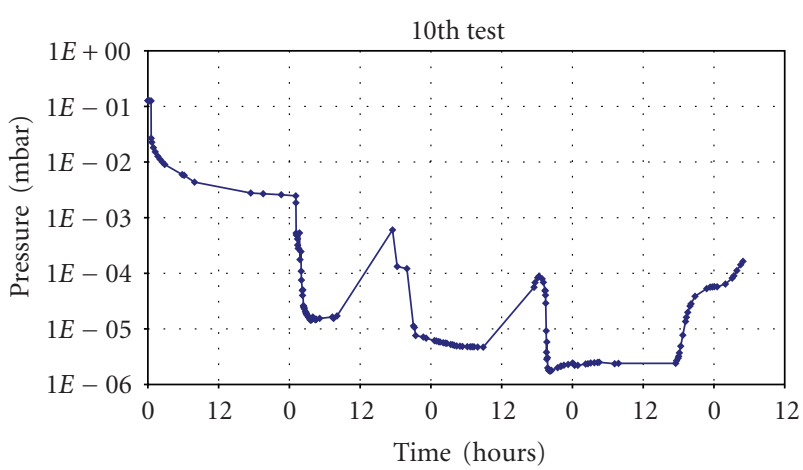

$\multimap$ Pressure

FIGURE 9: Pressure graph of intermediate test of endurance. This graph refers to a different test respect to the previous. The first part is the pumping of the system with a turbomolecular (two stages) pump, the second part (after first 24 hours) is the cooling down phase. Intermediate bumps reflect the end of the nitrogen in the fuel tank (again night time).

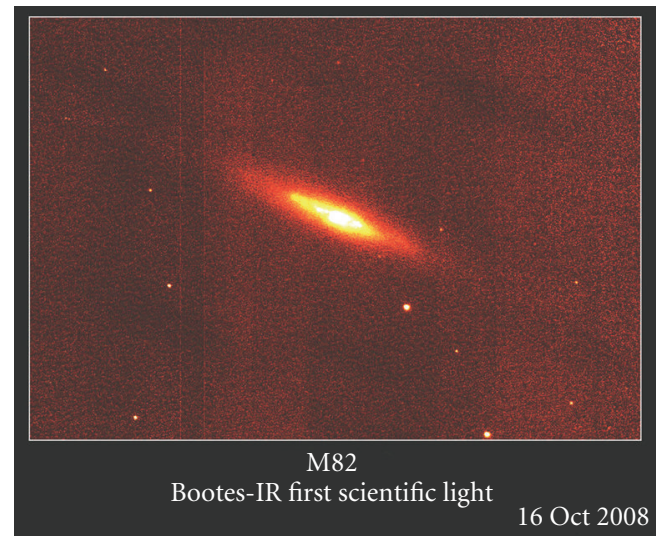

FIgURE 10: First scientific image of BIRCAM at BOOTES-IR telescope.

testing. During each phase of the project, we had the great opportunity of learning some important lessons about this kind of infrared camera for small telescope.

Most obvious and generically related to any astronomical instrument is that it is produced in a single unit (or at most a few). This fact marks a difference between astronomical instruments devices of mass production or from the prototype production. With the mass production, we concentrate on the statistics of possible failures and weak points of the series of machines. While building a prototype instead we concentrate more on enabling technologies more than on the functions of the final product.

If we look deep into BIRCAM project, we learned some lessons.

(i) Coordination between the telescope team and the camera team is essential in order to avoid unpredicted constraints (like for instance the space available between the telescope and the dome).

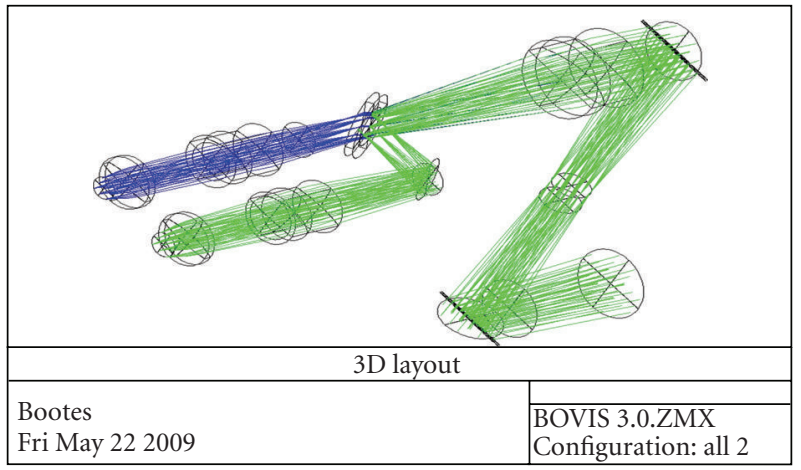

FIGURE 11: Schematic view of the future visible arm of BOOTES-IR.

(ii) The system engineering of the project must be kept into a scientific institution and not outsourced to industry. These kind of instruments needs an adequate amount of time dedicated to tests and optimizations.

(iii) Every constraint parameter must be carefully overdimensioned where possible. For instance, if the nitrogen consumption should be daily-based, the designer needs to assume some contingency parameter in dimensioning the tank capacity (i.e., doubled).

In particular, electronic subsystem problems taught us the following:

(i) Due to the lack of the science detector, a specific work package must be allocated for the tuning of bias and offset values of the electronic acquiring system.

(ii) The cabling is essentially fragile and it implies a great care during each operation of mounting and dismounting of the camera; on the other hand the thermal mass of the cabling induces a mechanical constraint on the deploy of the cables, increasing the probability to make some dangerous bends in the cables themselves. Specific care must be kept in the design phase, introducing where possible the option to disconnect cables between the dewar and the detector board.

(iii) Due to the complexity of windows-based and Linuxbased systems, specific attention must be kept in design the software. Where possible, a dedicated team should simulate and optimize the acquisition process in both systems.

\section{The Optical Arm}

Our team is now working to the new instrument that completes the BOOTES-IR telescope. As its "cousin" REM, this telescope has a visible arm [8]. The aim of this arm is to extend the field of monitoring at the visible range of the GRB afterglow. The visible arm will be fed by the transmission of the Dichroic in Figure 1. A preliminary study of the arm foresee a fold mirror that drives the light into a collimator. This collimator produce a beam that can be 
split into two arms through another dichroic that can divide the light into two arms, a $B+V$ and a $R$. In the conceptual study, these two arms are foreseen to hit the same detector, a CCD $2 \mathrm{k} \times 4 \mathrm{k}$ like the commercial ones, into two quadrants following previous studies [9].

The cost evaluation and the optimization of the optical elements is now argument of study of the Italo-Spanish working group.

\section{Conclusions}

The conclusion of this phase of the project is the beginning of the next phase. We strongly believe that such instrumentation require a lot of care and needs a strong team well coordinated. The next step stands in giving to the Spanish Astronomical Community, and more in general to the Astronomical community a new modern and powerful observing facility, possibly joined to the already existing network of the GRB community, and the robotic and remote telescopes community.

\section{Acknowledgments}

The authors need to make a special thank to the collaborators that physically made the camera: M. Canetti and A. Battocchio from RIALVACUUM, M. Gemma and A. Carbone from SKYTECH, G. Colombo and all their collaborators; the REM team for the filters providing, Favio Bortoletto for his valuable and decisive support in solving big electronic troubles. The authors thank F. Aceituno, C. Cárdenas, S. Castillo, J. Gorosabel, S. Guziy, P. Kubánek, T. Mateo Sanguino, J. C. del Toro and last but not least A. de Ugarte Postigo for the support during various phase of manufacturing and testing. This development was supported by Spanish Ministry of Science and Technology's projects AYA 2004-01515 and AYA 2007-63677.

\section{References}

[1] F. M. Zerbi, G. Chincarini, G. Ghisellini, et al., "The REM telescope: detecting the near infra-red counterparts of Gamma-Ray Bursts and the prompt behavior of their optical continuum," Astronomische Nachrichten, vol. 322, no. 5-6, pp. 275-285, 2001.

[2] S. Covino, F. M. Zerbi, G. Chincarini, et al., "The multifrequency robotic facility REM: first results," Astronomische Nachrichten, vol. 325, no. 6-8, pp. 543-548, 2004.

[3] F. M. Zerbi, G. Chincarini, G. Ghisellini, et al., "The REM telescope: detecting the near infra-red counterparts of Gamma-Ray Bursts and the prompt behavior of their optical continuum," Astronomische Nachrichten, vol. 322, no. 5-6, pp. 275-285, 2001.

[4] N. Gehrels, G. Chincarini, P. Giommi, et al., "The Swift Gamma-Ray burst mission," Astrophysical Journal, vol. 611, no. 2, pp. 1005-1020, 2004.

[5] J. S. Bloom, et al., "Autonomous observing and control systems for PAIRITEL, a $1.3 \mathrm{~m}$ infrared imaging telescope," in Astronomical Data Analysis Software and Systems XV, vol. 351 of ASP Conference Series, p. 751, San Francisco, Calif, USA, 2006.

[6] J. Greiner, W. Bornemann, C. Clemens, et al., "GROND-a 7channel imager," Publications of the Astronomical Society of the Pacific, vol. 120, no. 866, pp. 405-424, 2008.
[7] F. Vitali, F. M. Zerbi, G. Chincarini, et al., "The REM-IR camera: high quality near infrared imaging with a small robotic telescope," in Instrument Design and Performance for Optical/Infrared Ground-based Telescopes, vol. 4841 of Proceedings of SPIE, pp. 627-638, 2002.

[8] G. Tosti, M. Bagaglia, C. Campeggi, et al., "The REM optical slitless spectrograph (ROSS)," in Ground-Based Instrumentation for Astronomy, vol. 5492 of Proceedings of SPIE, pp. 689-700, June 2004.

[9] A. Riva and P. Spanò, "Compact multi-band visible camera for 1m-class fast telescopes," in Ground-Based and Airborne Instrumentation for Astronomy II, vol. 7014 of Proceedings of SPIE, pp. 1-9, Marseille, France, June 2008. 

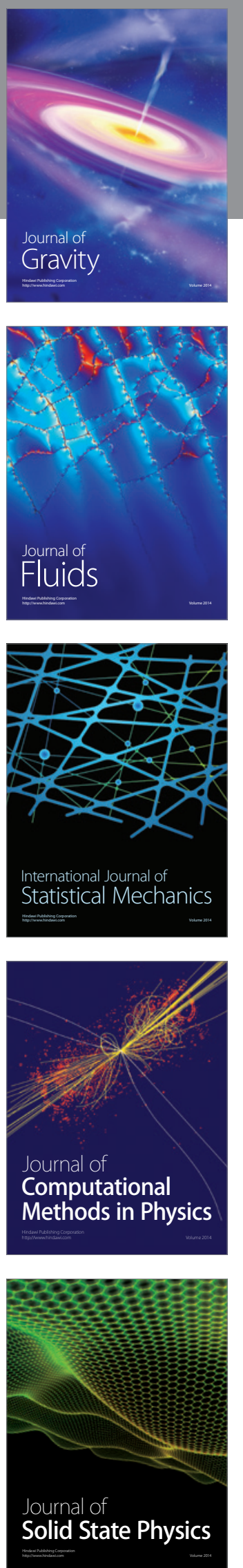

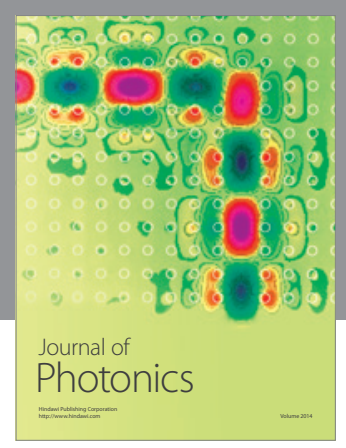

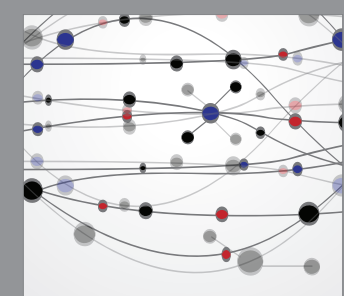

The Scientific World Journal
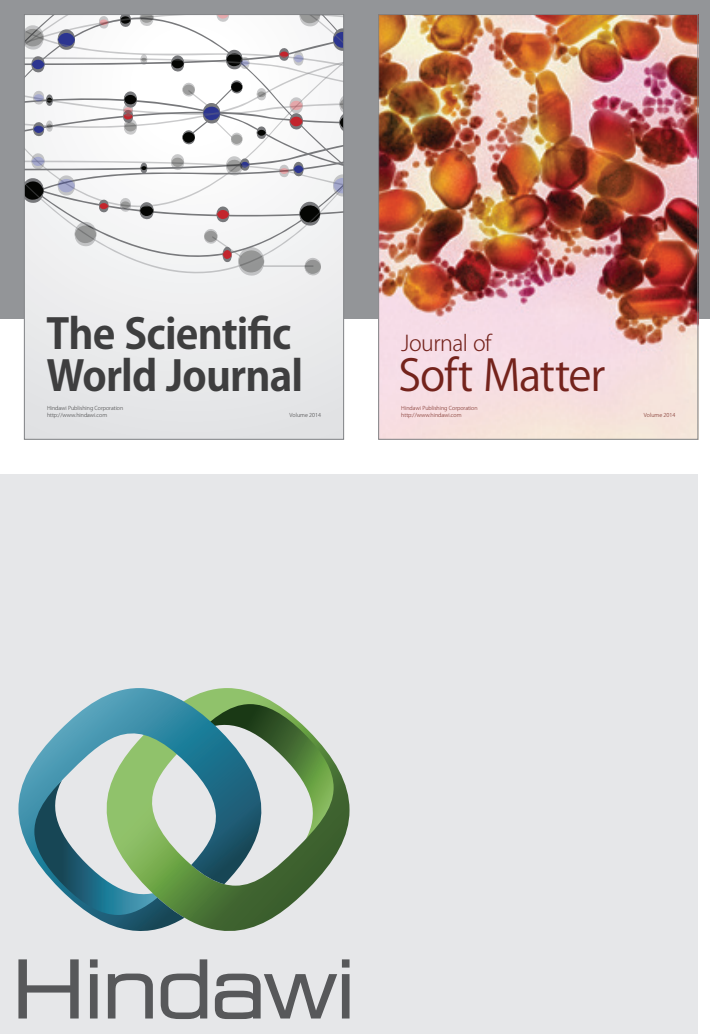

Submit your manuscripts at

http://www.hindawi.com
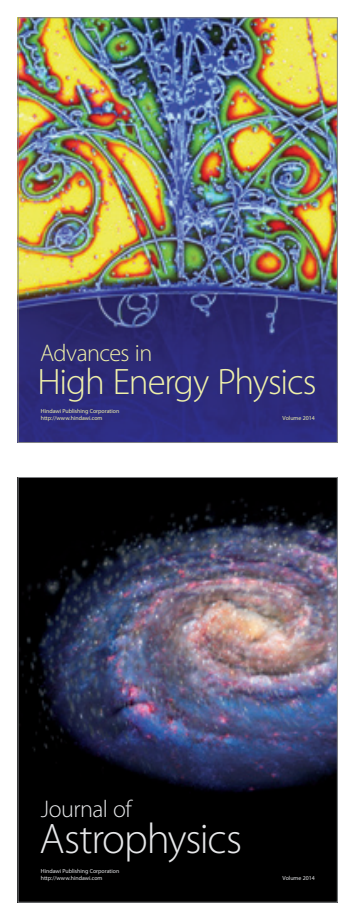
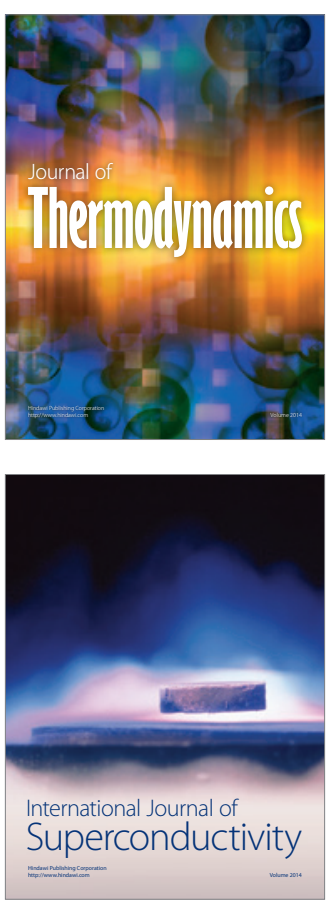
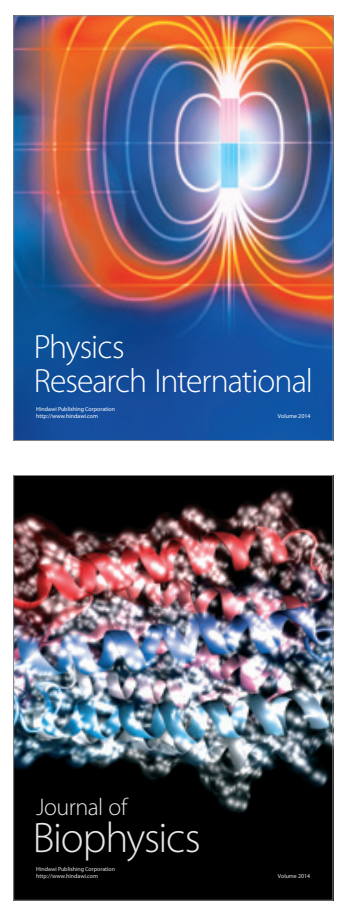
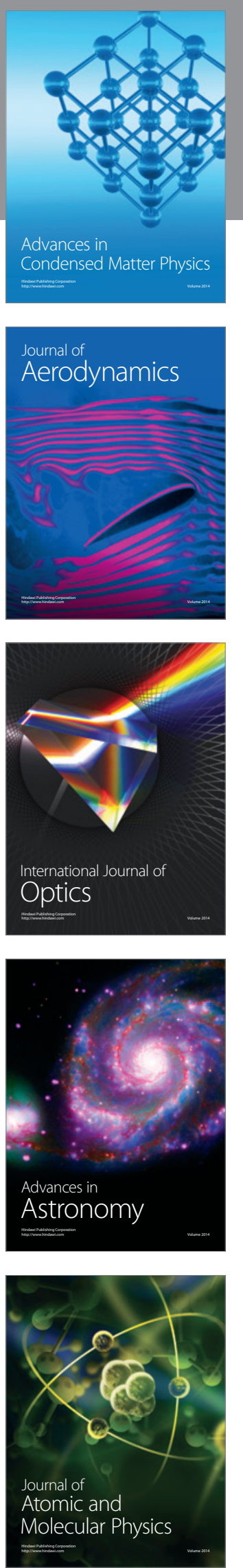